Ebisu Ebisu

Études japonaises Études japonaises

47 | printemps-été 2012

Catastrophes du 11 mars 2011, désastre de

Fukushima : fractures et émergences

\title{
Le déluge sans le Japon : aléa naturel et catastrophe dans la culture japonaise
}

The Flood Missed Japan: Natural Disasters Within Japanese Culture

日本を除く洪水一日本文化内部における自然の不確実性と桨害

Philippe Pelletier

CpenEdition

Journals

Édition électronique

URL : http://journals.openedition.org/ebisu/493

DOI : 10.4000/ebisu.493

ISSN : 2189-1893

Éditeur :

Institut français de recherche sur le Japon (UMIFRE 19 MAEE-CNRS), Maison franco-japonaise

Édition imprimée

Date de publication : 1 juin 2012

Pagination : 243-254

ISSN : $1340-3656$

Référence électronique

Philippe Pelletier, «Le déluge sans le Japon : aléa naturel et catastrophe dans la culture japonaise », Ebisu [En ligne], 47 | printemps-été 2012, mis en ligne le 03 avril 2014, consulté le 02 mai 2019. URL: http://journals.openedition.org/ebisu/493; DOI : 10.4000/ebisu.493 


\title{
Le déluge sans le Japon \\ Aléa naturel et catastrophe dans la culture japonaise
}

\author{
Philippe PELLETIER
}

Les nombreux aléas naturels qui touchent le Japon auraient pu structurer une philosophie centrée sur la catastrophe et son interprétation, axée sur une eschatologie, un millénarisme et des prophètes. Ces éléments existent dans la socioculture japonaise, mais ils ne sont pas dominants. Comme les supposés " harmonie avec la nature ", " absence de ressources naturelles " ou bien " manque d'espace ", ils sont doublement variables : dans l'espacetemps, et dans la perception qu'en ont Japonais et non-Japonais au sein d'un processus narratif qui s'entretient mutuellement (Pelletier 2012).

L'un de ces thèmes est celui du déluge, avec son corollaire l'engloutissement de l'île, qui, à la suite du tsunami du 11 mars 2011, ont été présentés par les médias français comme étant une obsession dans la tradition japonaise. Une réflexion géographique de temps long permet, sans prétention à l'exhaustivité, de faire une mise au point, en particulier à partir du cas de l'archipel des Gotō (Gotō rettō 五島列島) et de la légende de Kōrai-jima (Körai-jima densetsu 高麗島伝説).

Philippe Pelletier, géographe et diplômé en langue et civilisation japonaises, est professeur à l'université Lyon 2. Ses travaux sur le Japon portent actuellement sur l'insularité, l'iléité et les questions environnementales. 


\section{Du mappō à Komatsu}

L'eschatologie en tant que concept n'existe pas clairement dans la tradition socioculturelle japonaise dont le substrat repose sur le shintô. Son expression la plus visible n'apparaît qu'à la fin du XI ${ }^{\mathrm{e}}$ siècle, avec l'idée bouddhique $\mathrm{du}$ « monde du mappō» (mappō no yo 末法の世), ou le «monde de la règle finale ". L'histoire du monde se diviserait ainsi en trois périodes après que le Bouddha soit parvenu au nirvana, la troisième étant celle d'une dégénérescence où le peuple s'éloigne du Bouddha et n'atteint pas l'illumination. Dans son fameux Hōjōki 方丈記 (1212), l'ermite Kamo no Chōmei 鴨長明 se focalise sur les désastres naturels pour développer l'idée du mappō et celle du mujo 無情 (impermanence). Le grand incendie de Kyoto en 1177, le typhon de 1180 et le séisme de 1185 auraient suscité sa réflexion, sinon encouragé la croyance, mais le grand tsunami de 869 est déjà loin.

L'idée du mappō ne se propage donc au Japon que quatre siècles après l'introduction du bouddhisme. Elle correspond surtout à une période de troubles socio-économiques et politiques variés, et ne semble pas en rapport exclusif avec les aléas naturels. La suite des événements confirme cette perspective plus historique que géographique puisqu'une poignée de grands penseurs bouddhistes comme Hōnen 法然, Shinran 親鸞 ou Nichiren 日蓮 s'oppose à l'eschatologie du mappō en encourageant, sauf Nichiren, les fidèles à se sauver par la "puissance de l'autre "(tariki 他力), la grâce du Bouddha Amida. Ils réussissent à faire reculer la croyance catastrophiste, tandis que les aléas gardent leur rythme tellurique ou climatique. Les éruptions volcaniques, régulières et parfois dévastatrices, engendrent des cultes locaux, mais ne structurent pas de récits eschatologiques forts. C'est plutôt le sentiment de coexistence avec le volcan qui l'emporte (Augendre 2008).

Dix siècles plus tard, le processus semble se reproduire. Le livrecatastrophe de Komatsu Sakyō 小松左京 (1931-2011), La Submersion du Japon (Nippon chinbotsu 日本沈没, 1973), un best-seller, et les deux films éponymes $(1973,2006)$ apparaissent comme représentatifs d'une fantasmagorie traditionnelle ${ }^{1}$. L'éruption du mont Fuji 富士, métonymie du pays

1. Le fait que le premier film ait rapidement fait l'objet d'un remake aux États-Unis (Tidal Wave, 1975) confirme un tropisme occidental qui dépasse le cas japonais pour renvoyer à l'eschatologie biblique du Déluge. 
entier, qu'ils imaginent à l'origine de la catastrophe, figure aussi dans le sixième des huit contes oniriques de Rêve (Yume 夢, 1990), film de Kurosawa Akira 黒澤明, ici causée par une catastrophe nucléaire.

Komatsu fait partie de la génération qui a vécu la guerre de plein fouet. Son roman de science-fiction s'insère dans ce cycle de récits littéraires, cinématographiques ou dessinés, tels que Godzilla ou Akira, qui constituent une allégorie cathartique du Japon défait, détruit par la guerre et atomisé (Napier 1993). Alors que le cinéma de la Nouvelle vague japonaise exprime au même moment la contestation politisée d'une jeunesse japonaise et critique ouvertement la responsabilité belliciste de ses aînés, que ce soient les films réflexifs d'Ōshima Nagisa 大島渚 ou bien Sasori さそり (Scorpion), la série baroque d'Itō Shun.ya 伊藤俊也, La Submersion du Japon, de facture plus " populaire ", attire l'audience en jouant sur l'évocation mélancolique de la société japonaise traditionnelle. Comme le résume Susan Napier, ce film est " essentiellement une élégie du Japon perdu " (Napier 1993 : 335).

Or ce genre catastrophiste n'existe pratiquement pas avant la défaite de 1945. Il ne correspond pas aux "traités sur la ruine du pays Japon " (Nippon bōkoku-ron 日本亡国論), dont l'origine est chinoise et qui, depuis le début $\mathrm{du} \mathrm{xx}^{\mathrm{e}}$ siècle, évoluent sur le registre de la décadence du pays face à la modernisation et à l'occidentalisation. Il est essentiellement lié à la destruction atomique de Hiroshima et de Nagasaki en août 1945, explicitement évoquée dans la série des Godzilla. Avant de faire carrière dans la sciencefiction, Komatsu Sakyō est d'ailleurs un journaliste scientifique spécialisé dans les questions nucléaires.

Si l'on plonge plus loin dans le passé de la socioculture japonaise, jusqu'aux annales antiques du viII ${ }^{\mathrm{e}}$ siècle, on constate que celles-ci n'évoquent qu'indirectement le thème du déluge (Shibata 1969 ; Hall 1973 ; Ogiwara 1973 ; Tomiyama 1974). Le Kojiki 古事記 parle, dès ses premières lignes, d'une " terre qui venait de naître comme une tache d'huile flottante » et, selon le Nihon shoki 日本書紀 (version II), elle « ondoyait comme de l'huile flottante ". Mais rien n'indique d'où vient cette materia prima, cet océan primordial, si un cataclysme ou un déluge en est à l'origine. Comme la geste d'Izanagi-Izanami 伊邪那岐 • 伊邪那美 rappelle le mythe universel de l'Urmeer, les anthropoloques japonais Oka Masao 岡正雄 (1898-1982) et Ishida Eiichirō 石田英一郎 (1903-1968) l'ont rangée dans la catégorie du déluge. Mais la simple mention de l'océan primordial constituant un indice 
trop ténu, Oka suggéra que le récit japonais avait perdu son prélude. Il ne serait donc qu'une version tronquée des mythes austronésiens ou austroasiatiques racontant comment deux rescapés du déluge durent commettre un inceste pour repeupler la terre.

Mais, à la suite d'Ōbayashi Taryō 大林太良 (1929-2001), Alain Rocher dénonce cette extrapolation (Rocher 1997). Il évoque les mythes des Ryūkyū 琉球 qui racontent l'arrivée d'un couple incestueux par la mer sur une île qui n'est pas encore formée. En conséquence, «le thème du déluge ou du raz-de-marée peut être préfixé au récit, mais il n'est pas indispensable à la structure étiologique " (Rocher 1997 : 207). En revanche, l'inceste est le moteur de la structure narrative. Médiateur ambigu, il se présente à la fois comme l'équivalent du déluge et comme sa négation. C'est la même chose, car tous les deux rapprochent eau, hommes et bêtes, les hommes se nourrissant de viande crue puisqu'ils ont perdu le feu. «En d'autres termes, le déluge brouille les frontières entre les espèces comme l'espèce mélange la parenté et l'alliance" (Rocher 1997 : 208).

Mais ce n'est plus la même chose en ce sens que l'union interdite mais nécessaire permet de fonder un nouvel ordre. L'inceste est positif puisqu'il donne naissance à toute une progéniture, comprenant enfants, déités ou plantes nourricières. Et le déluge - ou son euphémisme, l'océan primordial - devient à son tour positif. Suivant cette logique, puisque les mythes austronésiens s’y réfèrent précisément, la mythologie japonaise, véhiculée par le premier peuplement de l'archipel japonais en provenance d'Asie du SudEst, aurait fait l'économie de la référence diluvienne. Elle se serait concentrée sur l'autre thème qui lui est lié, celui de l'inceste.

Les traditions d'Ishigaki-jima 石垣島, une île méridionale des Ryūkyū, constitueraient donc une exception. Elles racontent en effet que la submersion de la terre a été décidée par la divinité pour punir les hommes de leur discorde entre familles, n'épargnant qu'un jeune homme et sa sœur en vertu de leur piété (Rocher 1997 : 228, d'après Tanigawa Ken.ichi 谷川健一). Ce thème du châtiment divin et du déluge fait penser à la légende de Kōrai-jima dont l'anthropologue Yanagita Kunio 柳田国男 (1875-1962) en a retranscrit le récit pour la première fois, semble-t-il après l'avoir entendu sur un bateau qui l'emmenait dans le sud de l'archipel des Gotō en 1931 (Yanagita 1951). Mais cette légende est-elle spécifiquement japonaise? 


\section{La légende de Kōrai-jima, l'île engloutie}

Selon elle, la déité vénérée sur une île appelée Kōrai-jima apparaît dans le rêve d'un homme très pieux, et lui annonce que lorsque son visage se transformera il faudra que tous les habitants s'enfuient. L'homme en parle autour de lui, mais il est moqué de la plupart. Un beau jour, le visage de la déité devient rouge, les habitants sont surpris, et alors qu'ils s'enfuient précipitamment sur leurs bateaux, l'île est engloutie. Les rescapés s'échouent à Warabi-kojima 濒小島, un îlot situé tout au bord de la côte nord-orientale de Hisaka-jima 久賀島, l'une des îles de l'archipel des Gotō (Takahashi 2007).

Ce récit qui ressortit à la fable morale semble a priori facile à interpréter. D'après lui, il faut écouter les personnes pieuses, en contact avec les dieux, croire dans les rêves et les messages médiumniques; ceux qui ne le font pas sont punis ; enfin, la terre est fragile, la communauté insulaire aussi.

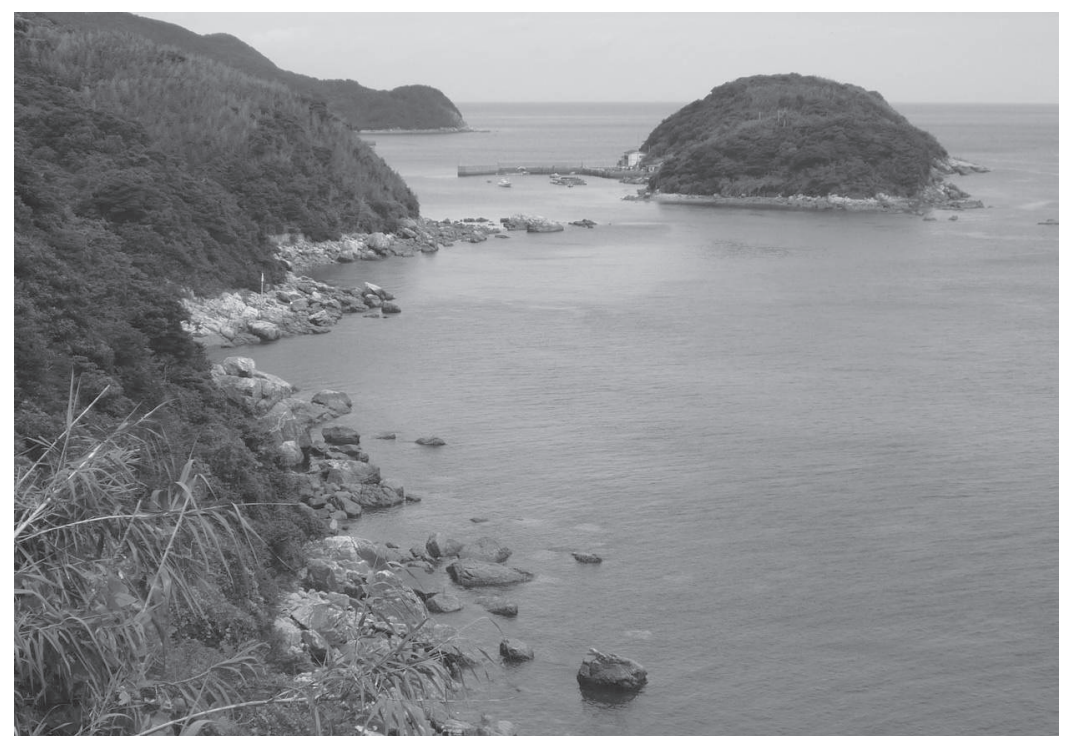

Lîlot de Warabi-kojima, au nord-est de lîle de Hisaka-jima, archipel des Gotō, Nagasaki-ken. C'est là, d'après la légende, que se seraient réfugiés les habitants de l'île engloutie de Kôrai-jima. @ Philippe Pelletier. 


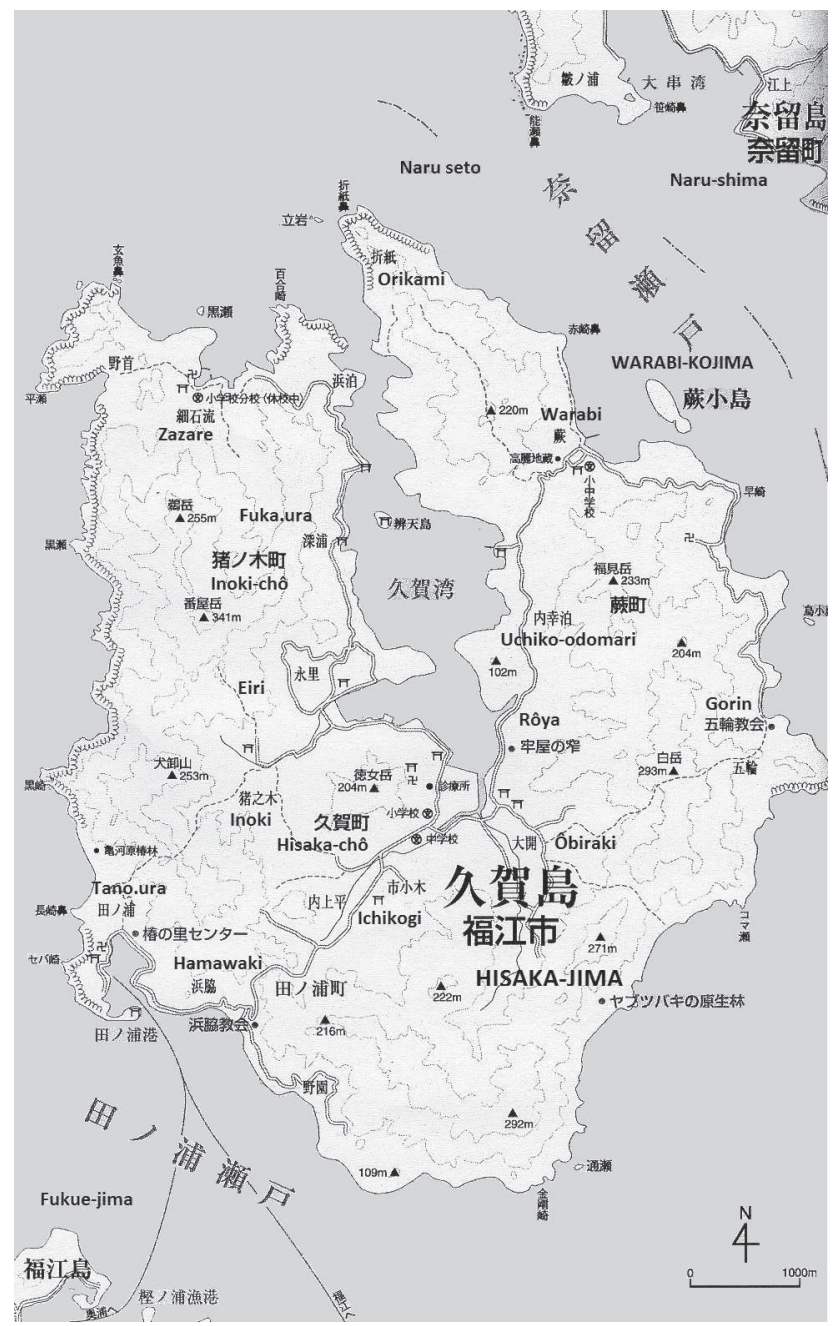

Carte de Hisaka-jima (Gotō rettō) avec, au nord-est, Warabikojima, îlot où se seraient réfugiés les rescapés de Kōrai-jima. Source : Nihon ritō sentā (2004). 
Mais il pose un problème : il ne nous dit rien sur le nom ou la nature de la déité en question, contrairement à tous les récits mythologiques japonais. Seuls ceux qui croient en elle semblent savoir de qui il s'agit, mais comment la vénérer si l'on ne connaît pas son nom, si l'on ne sait pas quelle place elle occupe dans le panthéon?

Une interprétation rapproche sémantiquement Kōrai de Hōrai 蓬莱, alias Penglai en chinois, l'île mythique que le taoïsme situe dans la mer orientale, où résideraient les bienheureux ayant découvert le secret de l'immortalité et que des héros intrépides chercheraient à gagner. Cette croyance chinoise fut mélangée avec la légende japonaise d'Urashima Tarō 浦島太郎, formalisée au Xve siècle et déjà présente dans le Man.yoshhü 万葉集 (chant [chōka 長歌] $\mathrm{n}^{\circ} 1740$ ). Des traces plus anciennes remontent au Nihon shoki par exemple (Pigeot \& Kosugi 1993). Leurs traits communs sont néanmoins peu nombreux, très généraux et, surtout, il manque des points essentiels.

La légende de Kōrai-jima ressemble bien davantage à un épisode du "Récit des commencements de la Terre et du Ciel " (Tenchi hajimari no koto 天地始之事), une version orale de la Bible secrètement transmise par les chrétiens cachés (kakure kirishitan かくれキリシタン) après l'interdiction et la répression du christianisme au début du XvII ${ }^{\mathrm{e}}$ siècle. Elle a été retranscrite au début du XIX ${ }^{e}$ siècle, et retrouvée par hasard en 1865 (Antille 2007 ; Bohner 1938 ; Proust 1997). Supprimant des passages importants de l'Ancien comme du Nouveau testament, elle introduit des croyances locales, shintô, bouddhistes ou confucéennes. Ces choix révèlent ce qui a frappé la communauté des chrétiens cachés ainsi que leur enracinement dans un endroit - une géographie - et une période - le martyr, le secret, la réapparition - sans dépendre d'une quelconque révélation divine, ou d'une catéchèse orthodoxe. Ce récit traduit la force des lieux, ici les petites îles, et l'archipel des Gotō.

Son troisième chapitre évoque une forte augmentation de la population poussant les habitants au vol'. Mécontent de la "cupidité » (yokuよく) et de “l'inclinaison au mal »( aku ni katamuki 悪にかたむき) qui se propagent, Dieu (Deusu 天帝) fait un oracle au souverain du royaume, dénommé Pappamaruji ぱつぱ丸次, une référence au pape des catholiques (pappa

2. “Hito ōku naru ni shitagaite, mina nusumi narai » 人多くなるにしたがいて、みな盗みな らい. 
ぱつぱ) et aux martyrs (maruchiru マルチル). D’après lui, « quand les yeux du shishigoma 獅子駒 deviendront rouges, le monde sera détruit par un tsunami 津波». Le shishigoma est un animal hybride, situé entre dragon, lion et chien, dont la statue, bien familière, monte la garde à l'entrée des sanctuaires. Pappamaruji se rend alors tous les jours au sanctuaire pour le vénérer. Des enfants se moquent de lui, estimant qu' "il est impensable qu'une “île de dix mille lieues" (Manrigashima 万里が島) puisse être détruite (metsubō めつ亡) comme cela », et ils décident de peindre en rouge les yeux du shishigoma.

Du coup, Pappamaruji fait monter précipitamment ses six enfants dans un kuribune くり船 (sorte de canoë construit à partir d'un rondin évidé), qu'il a préparé en prévision, tout en abandonnant son fils aîné qui « ne tient pas sur ses jambes ". Aussitôt, une "grande vague» (ónami 大浪) recouvre terre et ciel. Puis le shishigoma rejoint la troupe en portant le fils aîné sur son dos. Tous se réfugient en ramant sur une île, Ariō-shima あり王島. Quant aux « milliers de personnes englouties par la vague», elles tombent dans le Bembō ベんぼう, " l’un des enfers du monde précédent », soit les Limbes (limbo en portugais, la langue des visiteurs chrétiens au Japon).

\section{Warabi-kojima, alias Ariō-shima, l'île des rescapés}

Ce passage des "Commencements " retrace bien entendu l'épisode du Déluge et de l'Arche de Noé décrit par la Bible (Genèse, 6-7), mais en le déformant. Une comparaison entre les deux textes et la légende de Kōraijima, dégage des points communs et des différences.

Le récit japonais ne précise pas l'endroit exact où se déroule l'épisode du Déluge, mais comme c'est à ce moment qu'apparaît pour la première fois le personnage de Pappamaruji calqué sur une réalité historique, on en déduit qu'il s'agit d'un lieu terrestre. Cette hypothèse est renforcée par le fait que l'épisode suit la description cosmogonique ayant mis en scène Adam et Ėve - donc à l'échelle macro (le cosmos, le monde) - et qu'il passe brusquement à un autre niveau - à l'échelle micro (une communauté).

Cette communauté, que peut-elle être ? Vu le périmètre évoqué qui comporte un souverain, un temple (tera 寺, en l'occurrence confondu avec un sanctuaire shintô), quelques enfants (tenarai kodomo 手習子供), la mer, les bateaux et l'île (kono shimaこのしま), il s'agit d'une communauté insulaire, 
au moins allégoriquement. Elle correspondrait à Kōrai-jima, même si ce toponyme n'apparaît pas dans le texte japonais des "Commencements ».

La pluie biblique qui tombe sans discontinuité dans la légende de Kōraijima n'apparaît pas dans les "Commencements ». Le grand bateau de l'Arche devient un petit canoë avec " planches " (ita 板) et " cuillères " (shakushi Lゃ (し). Les animaux sont absents tandis que seuls les enfants de Pappamaruji sont évoqués, six au lieu de trois, et sans les femmes. On peut se demander si l'aîné, infirme, que Pappamaruji doit abandonner ne correspond pas à Hiruko 水蛭子, cet avorton-sangsue issu de la première copulation du couple divin Izanagi-Izanami, et que celui-ci abandonne aux flots sur un esquif de roseaux (ou de camphrier selon le Nihon shoki), mythème qui fait évidemment penser à l'épisode biblique de Moïse (Watanabe 2002).

La principale spécificité de la version japonaise du Déluge dans les "Commencements » est double: d'une part, l'apparition du shishigoma avec ses yeux rouges et, d'autre part, le rôle du milieu insulaire et maritime avec la "grande vague " (ōnami 大浪), et les îles désignées par leur toponyme comme Manriga-shima et Ariō-shima. Ces éléments puisent à d'autres sources. Les légendes chinoises ou coréennes décrivent ainsi des villes qui disparaissent sous les flots, mais pas dans la mer : dans des lacs (Lewis 2006). Au Japon, les palais se retrouvent au fond de la mer, comme dans la légende d'Urashima Tarō, sans d'ailleurs qu'ils soient décrits précisément.

Selon les spécialistes japonais, le nom d'Ariō-shima, mot à mot "L'Île où il y a un Roi ", fait penser par sa prononciation au mont Ararat (Genèse, 8 : 5). Il pourrait également évoquer un personnage du Heike monogatari ( $\mathrm{xII}^{\mathrm{e}}$ siècle), un certain Ariō 有王 qui aurait accompagné le moine bouddhiste Shunkan 俊寬 lors de son exil politique dans une île au sud de Kyūshū.

D'après l'anthropologue Tanigawa Ken.ichi, il renvoie aussi au Niraikanai ニライカナイ des Ryūkyū, ce monde ultramarin d'où viendraient régulièrement déités et visiteurs. Le terme de Niraikanai lui-même n'est en effet que l'une des variations phonétiques possibles pour désigner ce monde, puisqu'on trouve aussi celle d'Arō-shima あろう島 qui pourrait se confondre avec Ariō-shima. En outre, le toponyme de Miiraku 三井楽, un bourg des Gotō, était autrefois prononcé Mimiraku : cette diction ressemble à Nirai, comme Yanagita Kunio l'a remarqué. La croyance du Niraikanai, typique des Ryūkyū, serait ainsi remontée jusque dans les Gotō. 
Comme l'a également relevé Yanagita, plusieurs traces témoignent de la légende d'une île engloutie dans les Gotō. Outre Warabi-kojima, où l'on trouve le récit de la déité au visage rougissant, Yanagita signale l'existence d'une stèle (jizō地蔵) appelée Kōrai-jizō 高麗地蔵 dans le village de Warabi, situé en face sur Hisaka-jima, ainsi qu'une source appelée " eau de Kōrai ». Ce Kōrai-jizō représente Ebisu 恵比寿, la déité de la mer et du commerce, de façon originale puisqu' elle est peinte en rouge. Tout en relevant le lien avec l'épisode de Noé, Yanagita remarque que le motif des visages rougissants se retrouve aussi dans une légende chinoise. Enfin, l'une des versions des Commencements évoque, à propos du sauvetage à la rame vers Ariōshima, qu' " encore aujourd'hui la course appelée pèron ペーろん est une répétition de cet épisode ». Elle fait allusion à une course nautique qui se déroule encore à Nagasaki 長崎 à la fin du mois de juillet.

Malgré ces récits et malgré ce qu'on pouvait attendre d'un peuple vivant historiquement sur un archipel battu par les flots ou les tsunami, la tradition japonaise est donc chiche de références au déluge. Il faut même que ce soient un récit inspiré de la Bible et une légende qui lui est très probablement connectée, comportant en outre des références chinoises, qui en parlent le mieux, tandis que la mythologie du Kojiki et du Nihon shoki se contente d'évoquer un océan primordial sans expliquer par quoi il a été provoqué.

L'allégorie du déluge au Japon semble donc relever davantage de circonstances historiques tragiques (le martyrologe chrétien, l'holocauste atomique) que d'un substrat géographique consubstantiellement hostile. 


\section{Bibliographie}

ANTILLE Géraldine, 2007

Les Chrétiens cachés du Japon. Traduction et commentaires des « Commencements

du Ciel et de la Terre ", Genève,

Labor et Fides.

AUGENDRE Marie, 2008

Vivre avec le volcan. Une géographie

du risque volcanique au Japon,

université Lyon 2, thèse, Ph. Pelletier dir.

\section{BOHNER Alfred, 1938}

«Tenchi Hajimari no Koto, wie Himmel und Erde entstanden », Monumenta Nipponica, 1-2 : 465-514.

\section{CHAMBERLAIN Basil Hall, 1973}

Ko-ji-ki, or Records of Ancient Matters, Tokyo, The Asiatic Society of Japan, rééd. 2005, Tuttle Publishing.

IWAKIRI Makoto 岩切誠, SHIMOZURU Daisuke 下鶴大輔 (dirs.), 2001

Nihon saigai-shi 日本災害史, Tokyo, Nihon tosho sentā 日本図書センター, 4 vol.

LEWIS Mark Edward, 2006

The Flood Myths of Early China, Albany, Suny Press.

NAPIER Susan J., 1993

«Panic Sites: the Japanese Imagination of Disaster from Godzilla to Akira », Journal of Japanese Studies, 19-2 : 327-351.
NIHON RITŌ SENTĀ 日本離島センター (éd.), 2004

Nihon no shima gaido - Shimadas 日本 の島ガイド シマダス (Shimadas. Le guide des îles japonaises), Tokyo, Nihon ritō sentā.

Kojiki. Jōdai kayō 古事記·上代歌謡 (Le Kojiki et les poèmes de l'Antiquité), 1973

in Nihon koten bungaku zenshū 日本 古典文学全集 (Collection complète de la littérature classique du Japon), t. 1, annoté et traduit par Ogiwara Asao 荻原 浅男 et Kōnosu Hayao 鴻巣隼雄, Tokyo, Shōgakukan 小学館.

\section{PELLETIER Philippe, 2012}

La fascination du Japon, Paris, Le Cavalier bleu.

PIGEOT Jacqueline

\& KOSUGI Keiko, 1993

Voyages en d'autres mondes. Récits japonais du Xvi siècle, Arles/Paris, Philippe Picquier/Bibliothèque Nationale.

PROUST Jacques, 1997

L'Europe au prisme du Japon (XVI XVIII ${ }^{e}$ siècle), entre humanisme, ContreRéforme et Lumières, Paris, Albin Michel.

SHIBATA Masumi \& Maryse, 1969 Le Kojiki. Chronique des choses anciennes, Paris, Maisonneuve et Larose. 
TAKAGI Ichinosuke 高木市之助 \& TOMIYAMA Tamizō 富山民蔵, 1974 Kojiki sōsakuin 古事記總索引 (Kojiki, index général), Tokyo, Heibonsha 平凡社.

TAKAHASHI Daisuke 高橋大輔, 2007 «Kōrai-jima densetsu no genba kentō : kaichū ni chinshita rakuen o motomete» 高麗島伝説の現場検討

海中に沈した楽園を求めて (Examen sur le terrain de la légende de Kōrai-jima : à la recherche du paradis englouti dans la mer), Fukkatsu no shima. Gotō Hisaka-jima kirisutokyō bohi chōsa hōkokusho 復活の島一一五島·久賀島キリ ス卜教墓碑調査報告書 (La Résurrection d'une île. Compte rendu d'une enquête sur les tombes chrétiennes de Hisakajima des Gotō), Nagasaki, Nagasaki Bunken-sha 長崎文献社, pp. 24-25.

WATANABE Kōji, 2002

"L'énigme de Hiruko dans la mythologie japonaise, le mythe de l'enfant-sangsue ou le mythe du jeune soleil ? », Iris, $23: 55-62$.

YANAGITA Kunio 柳田国男, 1951

«Kōrai-jima no densetsu » 高麗島の伝 説 (La légende de Kōrai-jima), Shima no jinsei 島の人生 (La Vie des îles), Yanagita Kunio zenshū 柳田国男全集, vol. 1, Tokyo, Sōgensha 創元社, pp. 387-410 [éd. originale 1933]. 